\title{
MicroRNA-34a inhibits cell growth and migration in human glioma cells via MMP-9
}

\author{
XUEPENG WANG ${ }^{1}$, XI CHEN $^{2}$, LIN SUN $^{3}$, XIAOLI BI $^{4}$, HAITAO HE $^{5}$, LEI CHEN $^{1}$ and JINFENG PANG ${ }^{1}$ \\ Departments of ${ }^{1}$ Neurosurgery, ${ }^{2}$ Neurology, ${ }^{3}$ Production, ${ }^{4} \mathrm{CT}$, and ${ }^{5} \mathrm{Cycle}$ of Internal, \\ Affiliated Hospital of Beihua University, Jilin City, Jilin 132000, P.R. China
}

Received February 15, 2018; Accepted January 21, 2019

DOI: $10.3892 / \mathrm{mmr} .2019 .10233$

\begin{abstract}
The present study was designed to investigate the function of matrix metalloproteinase-9 (MMP-9) in human glioma cells and the potential regulatory mechanisms. Reverse transcription-quantitative polymerase chain reaction was used to analyze the expression of MMP-9 and microRNA-34a (miR-34a) in the plasma of patients with glioma and healthy volunteers. MTT and Transwell assays were used to assess cell growth and migration, respectively. Annexin-V/propidium iodide staining was used to measure cell apoptosis. In addition, MMP-9 expression was measured using western blot analysis. In patients with glioma, MMP-9 expression was increased, while miR-34a expression was suppressed, compared with the normal group. Overall survival (OS) and disease-free survival (DFS) of patients with high MMP-9 expression were decreased compared with those with low MMP-9 expression. OS and DFS of patients with low miR-34a expression were decreased compared with those with high miR-34a expression. Downregulation of miR-34a promoted cell growth and migration, and inhibited apoptosis in U251-MG glioma cells. However, overexpression of miR-34a inhibited cell growth and migration, and induced apoptosis in glioma cells. Furthermore, downregulation of miR-34a using anti-miR-34a induced MMP-9 protein expression in glioma cells; whereas, overexpression of miR-34a suppressed MMP-9 protein expression in glioma cells. SB-3CT, an inhibitor of MMP-9, attenuated the effects of miR-34a mimic on glioma cells. Together, these results indicated that miR-34a inhibited cell growth and migration in human glioma cells by regulating MMP-9.
\end{abstract}

\section{Introduction}

Associated statistics indicate that the morbidity of primary central nervous system tumors is 20.59/100,000 (1). In

Correspondence to: Dr Jinfeng Pang, Department of Neurosurgery, Affiliated Hospital of Beihua University, 12 Liberation Road, Jilin City, Jilin 132000, P.R. China

E-mail: qatgwk5010399@126.com

Key words: matrix metalloproteinase-9, glioma, microRNA-34a, cell growth, migration addition, the 5-year and 10-year survival rates of patients with malignant central nervous system tumors are 33.8 and $28 \%$, respectively (2). Malignant glioma is incurable and accounts for $\sim 80 \%$ of all intracranial malignant tumors (2). Surgical resection of the tumor is not sufficient to achieve a cure. In certain cases, complete resection cannot be achieved due to loss of brain function. Therefore, the comprehensive treatment combination of surgery, radiotherapy and chemotherapy is most commonly adopted in the clinic at present (3). Nevertheless, therapeutic efficacy is still unsatisfactory (3). The field of molecular biology continues to be developed and applied in tumor research. Targeted molecular therapy is being used to overcome the difficulty of treating malignant tumors like glioma (1). Specifically, identifying the important genes regulating glioma proliferation and investigating their roles is of positive clinical significance. This study contributes to revealing the molecular mechanism of glioma proliferation, and therefore allows effective therapeutic means to be developed (4).

Glioma is the most common primary intracranial tumor in adults, and frequently exhibits a high malignancy grade, high possibility of recurrence and a poor prognosis. It has short median survival once it is diagnosed (5). Profound progress has been achieved in cancer treatment, including improvements in surgery, chemotherapy, radiotherapy and immunotherapy. However, the overall prognosis is not improved (5). Consequently, microRNAs (miRNA/miR), a class of endogenous non-coding small molecular RNA 18-24 nucleotides in length, has attracted attention from scientists. miRNAs can regulate target genes to alter the expression of target proteins (6). The clinical significance of microRNAs is in glioma diagnosis, chemotherapeutic efficacy evaluation, anti-angiogenesis, treatment and prognosis (6). An increasing number of studies have demonstrated that, miRNA serves a vital role in glioma tumorigenesis and development $(6,7)$. They may serve as a novel index for the clinical diagnosis and prognosis evaluation, and as new therapeutic targets for glioma in the future. miRNAs can act as oncogenes or tumor suppressor genes (7), and have complex biological functions (7). As a result, cancer heterogeneity at the genetic and epigenetic levels is of therapeutic significance. Furthermore, it also marks the challenge in the reasonable design of effective therapeutics (7).

Matrix metalloproteinases (MMPs) are a class of proteases that degrade the vascular basement membrane, and hydrolyze 
the extracellular matrix and other matrix components (5). MMPs have critical functions in promoting tumor invasion and angiogenesis (5). Currently, >20 MMPs have been discovered, which can be divided into four categories according to different substrates. The substrates include collagenases, gelatinases, matrix degradation enzymes and membrane MMPs. Multiple studies have demonstrated that high MMPs expression is correlated with metastasis and poor prognosis patients with epithelial tumor tissues, including lung cancer, gastric cancer, colon cancer, breast cancer and prostate cancer (8). The MMP gelatinases, namely MMP-2 and MMP-9, have molecular weights of 72 and $92 \mathrm{kDa}$, respectively (8). MMP-2 can degrade type IV collagen in the extracellular matrix, which promotes tumor cell diffusion (9). In addition, it can promote tumor invasion and metastasis. MMP-2 expression is gradually increased with the increased tumor, node and metastasis classification (9). Typically, the positive expression rate in patients with lymph node metastasis is increase compared with those with no lymph node metastasis (10). MMP-9 is the enzyme with the greatest molecular weight out of the MMPs. It is secreted in the form of a zymogen (10). Notably, it can hydrolyze the cell basement membrane, type IV and type V collagens in the extracellular matrix, and fibronectin components; thus, resulting in basement membrane destruction, allowing tumor cells to invade the connective tissue, small vessels and lymphatic vessels, resulting in metastasis (8). Jia et al (11) reported that miRNA-34a reduced the migration and invasion of tongue squamous cell carcinoma by targeting MMP-9 and MMP-14. Tabouret et al (12) showed that MMP2 and MMP9 serum levels are associated with favorable outcome in patients with inflammatory breast cancer. The present study aimed to investigate the function of MMP-9 in human glioma cells and its potential regulatory mechanisms.

\section{Materials and methods}

Clinical specimens. Peripheral blood $(5-10 \mathrm{ml})$ was obtained from patients with glioma $(n=82)$ following surgery and healthy volunteers $(n=42)$ at the Affiliated Hospital of Beihua University (Jilin City, China) between February 2010 and December 2014 (Table I). Peripheral blood was centrifuged at $1,000 \mathrm{x} \mathrm{g}$ for $10 \mathrm{~min}$ at $4^{\circ} \mathrm{C}$ and the serum was stored at $-80^{\circ} \mathrm{C}$ until analysis. The present study was approved by the Ethics Committee of Affiliated Hospital of Beihua University. The study was performed in accordance with the regulations of the Institutional Review Board of Affiliated Hospital of Beihua University. Written informed consent was obtained from all enrolled patients prior to surgery. Written informed consent was also obtained from healthy volunteers. The follow-up period for the patients was every three months by telephone for 5 years.

RNA extraction and miRNA reverse transcription-quantitative polymerase chain reaction ( $R T-q P C R)$. Total RNAs from the serum samples and cells were extracted using TRIzol reagent (Invitrogen; Thermo Fisher Scientific, Inc., Waltham MA, USA). Total RNAs was used to synthesize complementary DNA using SuperScript II Reverse Transcriptase (Invitrogen; Thermo Fisher Scientific, Inc.) at $37^{\circ} \mathrm{C}$ for $30 \mathrm{~min}$ and $84^{\circ} \mathrm{C}$ for $1 \mathrm{~min}$. qPCR was performed with StepOne Real-Time PCR System (Applied
Table I. Characteristics of glioma patients and healthy volunteers.

\begin{tabular}{lcc}
\hline Variables & $\begin{array}{c}\text { Patients } \\
(\mathrm{n}=82)\end{array}$ & $\begin{array}{c}\text { Healthy volunteers } \\
(\mathrm{n}=42)\end{array}$ \\
\hline Age (years) & & \\
$\leq 55$ & 40 & 19 \\
55 & 42 & 23 \\
Sex & & 17 \\
Female & 35 & 25 \\
Male & 47 & \\
Tumor size (cm) & & \\
$\leq 3.0$ & 15 & \\
$>3.0$ & 67 & \\
Edmondson grade & & \\
I & 7 & \\
II & 13 & \\
III-IV & 62 & \\
\hline
\end{tabular}

Biosystems; Thermo Fisher Scientific, Inc.) and SYBR Premix Ex $\mathrm{Taq}^{\mathrm{TM}}$ (Takara Bio, Inc., Otsu, Japan). miR-34a forward, 5'-CCA GCTGTGAGTGTTTCTTTG-3' and reverse, 5'-CAGCAC TTCTAGGGCAGTAT-3'; U6 forward, 5'-GCTTCGGCAGCA CATATACTAAAAT-3' and reverse, CTTCGGCAGCACATA TACGCTTCACGAATTTGCGTGTCAT-3'; MMP-9 forward, 5'-AGACCTGGGCATTCCAAAC-3' and reverse, 5'-CGGCAA GTCTTCCGAGTAGT-3'; reference gene ( $\beta 2$ microglobulin) forward, 5'-TACACTGAATTCACCCCCAC-3' and reverse, 5'-CATCCAATCCAAATGCGGCA-3'. The reaction conditions were pre-denaturation at $95^{\circ} \mathrm{C}$ for $10 \mathrm{~min}$, followed by 40 cycles of denaturation at $95^{\circ} \mathrm{C}$ for $5 \mathrm{~min}$, annealing at $60^{\circ} \mathrm{C}$ for $30 \mathrm{sec}$ and elongation at $72^{\circ} \mathrm{C}$ for $30 \mathrm{sec}$. The relative expression levels were calculated using the 2-Cq method (13). Low expression of MMP9 was $<2$ of the MMP9 expression in healthy volunteers, high expression of MMP9 was $>2$ of the MMP9 expression of healthy volunteers; low expression of miR-34a was $<2$ of miR-34a the expression of healthy volunteers, high expression of miR-34a was $>2$ of the miR-34a expression of healthy volunteers.

Cell lines, culture and transfection. The U251-MG human glioma cell line was purchased from the Cell Bank of Type Culture Collection of Chinese Academy of Sciences (Shanghai, China) and maintained in Dulbecco's modified Eagle's medium (DMEM; Gibco; Thermo Fisher Scientific, Inc.) supplemented with $10 \%$ fetal bovine serum (FBS; Gibco; Thermo Fisher Scientific, Inc.) and $1 \%$ antibiotic-antimycotic solution at $37^{\circ} \mathrm{C}$ in a humidified $5 \% \mathrm{CO}_{2}$. miRNA-34a (5'-CACCGGTTGTTG TGAGCAATAGTA-3' and 5'-AAACTACTATTGCTCACA ACAACC-3'), anti-miRNA-34a (5'-ACAACCAGCUAAGAC ACUGCCA-3' and 5'-TGACCGACATGTTCAGACA-3') and negative control mimics (5'-CCCCCCCCCCCCCCC-3' and 5'-CCCCCCCCCCCC-3') were purchased from Shanghai GenePharma Co., Ltd. (Shanghai, China). U251-MG cells $\left(1 \times 10^{6}\right.$ cells/well) were transfected with $100 \mathrm{ng}$ miRNA-34a, $100 \mathrm{ng}$ anti-miRNA-34a and $100 \mathrm{ng}$ negative control mimics using Lipofectamine ${ }^{\circledR} 3000$ (Invitrogen; Thermo Fisher 
Scientific, Inc.), according to the manufacturer's protocol. Following $4 \mathrm{~h}$ of transfection, SB-3CT (an inhibitor of MMP-9) was incubated with cells for $44 \mathrm{~h}$.

Luciferase assays. The 3'untranslated region (UTR) sequences of MMP-2 containing wide-type or mutant-type miR-34a binding sites were amplified by PCR into pGL3-control vectors (Promega Corporation). The constructed reporter vector (100 ng of MMP-2 plasmid and $100 \mathrm{ng}$ of miR-34a mimics) was cotransfected into $\mathrm{U} 251$ cells $\left(1 \times 10^{6}\right.$ cell $\left./ \mathrm{ml}\right)$ using Lipofectamine ${ }^{\circledR} 3000$ (Invitrogen; Thermo Fisher Scientific, Inc.). Luciferase activity was detected using dual-luciferase reporter assay system (Promega Corporation) after $48 \mathrm{~h}$. miR-34a was predicted to target the 3'-UTR of MMP-9 using TargetScan software version 7.1 (http://www.targetscan.org). Luciferase activity was normalized against Renilla luciferase.

Cell proliferation assay and LDH activity. The cells (1x10 $/$ well) were seeded in 96-well plates and transfected with Lipofectamine ${ }^{\circledR} 2000$ (Invitrogen; Thermo Fisher Scientific, Inc.). MTT $(20 \mu \mathrm{l})$ was added into each well and incubated for $4 \mathrm{~h}$ at $37^{\circ} \mathrm{C}$. A total of $150 \mu \mathrm{l}$ isopropanol was added and the cells were incubated at room temperature in the dark for $20 \mathrm{~min}$. The absorbance was measured using a microplate spectrophotometer (Bio-Tek Instruments, Inc., Winooski, VT, USA) at $492 \mathrm{~nm}$.

LDH activity was measured using LDH activity kits (C0016; Beyotime Institute of Biotechnology, Haimen, China) and the absorbance was measured using a microplate spectrophotometer (Bio-Tek Instruments, Inc.) at $450 \mathrm{~nm}$.

Transwell assay. Cells ( $2 \times 10^{4}$ cells) were seeded into the upper chambers of Transwell chambers in a 24 well plate (Corning Incorporated, Corning, NY, USA) with DMEM and $500 \mu 1$ DMEM supplemented with 10\% FBS was added into the lower wells as the chemo-attractant. Following cultivation for $48 \mathrm{~h}$, the filters were fixed with $4 \%$ paraformaldehyde for $15 \mathrm{~min}$ and stained with $5 \%$ crystal violet for $10 \mathrm{~min}$ at room temperature. Laser scanning confocal microscopy (Leica Microsystems GmbH, Wetzlar, Germany) was used for cell observation.

Cell apoptosis assay. Cells were washed with PBS and harvested by centrifugation at $1,000 \mathrm{x} \mathrm{g}$ for $10 \mathrm{~min}$ at room temperature. Cells were stained with $5 \mu 1$ Annexin V (allophycocyanin) and $5 \mu \mathrm{l}$ propidium iodide (BD Biosciences, San Jose, CA, USA) for $15 \mathrm{~min}$ at room temperature in the dark. The apoptosis rate was acquired with a fluorescence-activated cell sorting Canto II flow cytometer (BD Biosciences) and analyzed using FlowJo 7.6.1 (FlowJo,LLC, Ashland, OR, USA).

Caspase-3/9 activity levels. Cellular nuclear protein was extracted using a RIPA buffer (Beyotime Institute of Biotechnology) and the protein concentration was detected using a bicinchoninic acid kit (Beyotime Institute of Biotechnology). A total of $10 \mu \mathrm{g}$ of protein was used to measure the caspase-3/9 activity levels using caspase-3/9 activity levels kits (cat. nos. C1115 and C1158; Beyotime Institute of Biotechnology). The absorbance was measured using a microplate spectrophotometer (Bio-Tek Instruments, Inc.) at $405 \mathrm{~nm}$.
Western blot analysis. Cellular nuclear protein was extracted using a RIPA buffer (Beyotime Institute of Biotechnology) and the protein concentration was detected using a bicinchoninic acid kit (Beyotime Institute of Biotechnology). A total of $50 \mu \mathrm{g}$ of protein loaded in each well and separated using $10 \%$ SDS-PAGE gel and transferred onto polyvinylidene difluoride membranes (EMD Millipore, Billerica, USA). Membranes were blocked with $5 \%$ skim milk in TBS with $0.1 \%$ Tween-20 (TBST) for $2 \mathrm{~h}$ at room temperature and incubated with primary antibodies against MMP-9 (cat. no. sc-12759; 1:1,000; Santa Cruz Biotechnology, Inc.) and GAPDH (cat. no. sc-51631; 1:5,000; Santa Cruz Biotechnology, Inc.) overnight at $4^{\circ} \mathrm{C}$. Membranes were washed with TBST for $15 \mathrm{~min}$ and incubated with the corresponding horseradish peroxidase (HRP)-conjugated secondary antibody (cat. no. A0208; Beyotime Institute of Biotechnology; 1:1,000) for $1 \mathrm{~h}$ at room temperature. Membranes were visualized using a Millipore Enhanced Chemiluminescence system (EMD Millipore) and analyzed using Image Lab 3.0 (Bio-Rad Laboratories, Inc., Hercules, CA, USA).

Immunofluorescence. Cells ( $1 \times 10^{5}$ cells/well) were washed with PBS and fixed in $4 \%$ paraformaldehyde at $4^{\circ} \mathrm{C}$ for $15 \mathrm{~min}$ at room temperature. Cells were blocked with $5 \%$ bovine serum albumin (Beyotime Institute of Biotechnology) and 0.25 Triton X-100 for $1 \mathrm{~h}$ at room temperature. Cells were incubated with MMP-9 antibody (cat. no. sc-12759; 1:1,000; Santa Cruz Biotechnology, Inc.) at $4^{\circ} \mathrm{C}$ overnight. Cells were washed with PBS $0.1 \%$ Tween 20 and then goat anti-rabbit IgG-CFL 555 (cat. no. sc-362272; 1:1,000; Santa Cruz Biotechnology, Inc.) were used for $1 \mathrm{~h}$ at $37^{\circ} \mathrm{C}$. Cells were stained with DAPI $(5 \mathrm{mg} / \mathrm{ml})$ for $15 \mathrm{~min}$ in darkness at room temperature and washed with PBST $(0.1 \%$ Tween-20) for $15 \mathrm{~min}$. Laser scanning confocal microscopy (Leica Microsystems $\mathrm{GmbH}$ ) was used for cell observation and analysis was performed using Image Lab 3.0 (Bio-Rad Laboratories, Inc.).

Statistical analysis. The data are expressed as the mean + standard deviation. Kaplan-Meier analysis and a log-rank test were used to evaluate the effects of MMP-9 or miRNA-34a on the overall survival (OS) and disease-free survival (DFS) of patients with glioma. The differences between different groups were compared using Student's t-tests or one-way analysis of variance and Tukey's post-hoc test. $\mathrm{P}<0.05$ was considered to indicate a statistically significant difference.

\section{Results}

miR-34a and MMP-9 expression. To investigate the role of miR-34a and MMP-9 expression in glioma, the expression of miR-98 in serum from patients with glioma was examined using RT-qPCR. As presented in Fig. 1A, MMP-9 expression was significantly elevated in serum from patients with glioma compared with the normal group $(\mathrm{P}<0.01)$. While miR-34a expression was significantly lower in serum from patients with glioma compared with the normal group ( $\mathrm{P}<0.01$; Fig. 1B). These results revealed that miR-34a and MMP-9 may be associated with glioma cell growth. Then, whether miR-34a and MMP-9 affected the OS and DFS of glioma patients was determined. As presented in Fig. 1C and D, the OS and DFS of patients with 

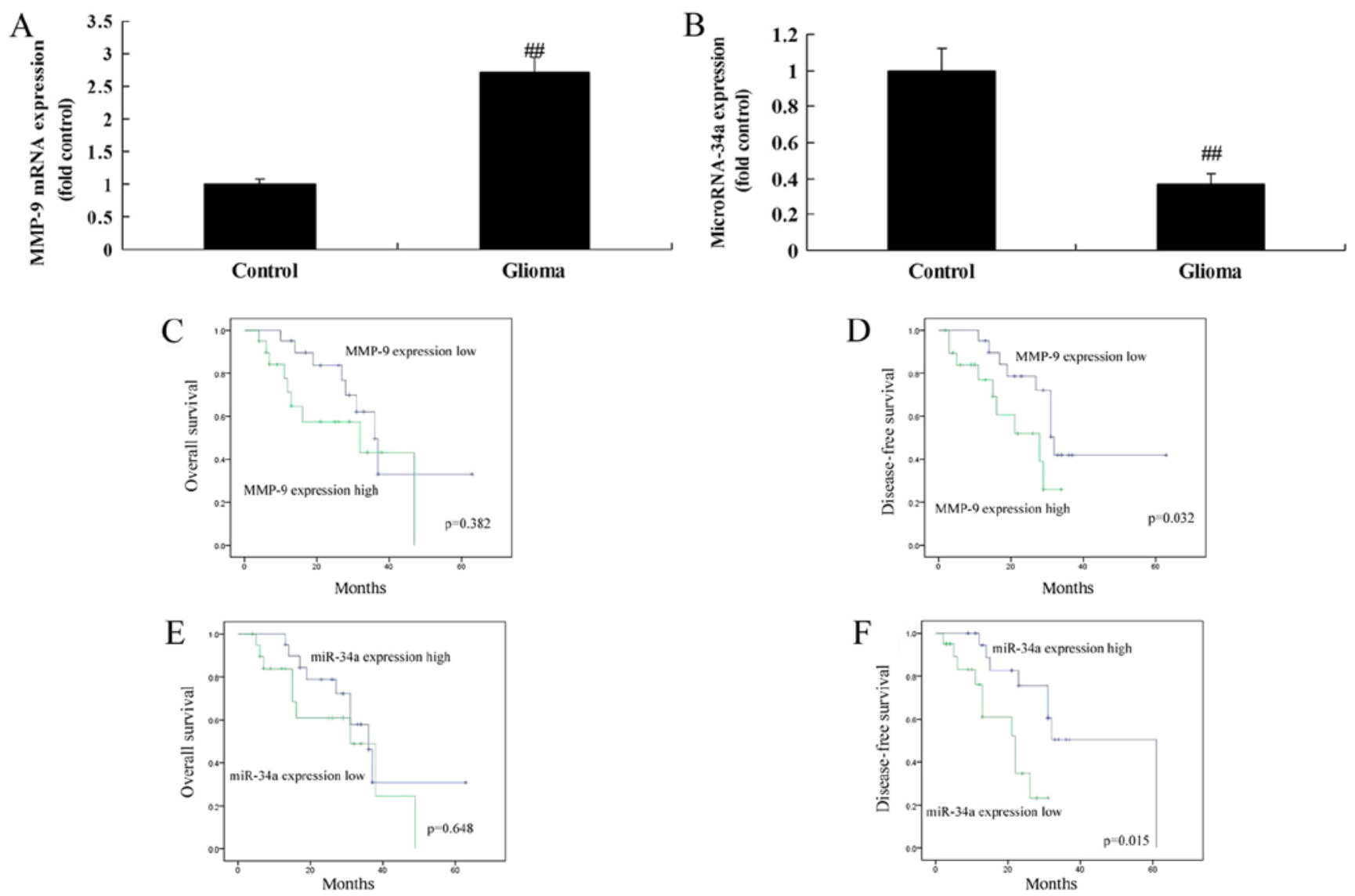

Figure 1. miR-34a and MMP-9 expression. (A) MMP-9 expression and (B) miR-34a expression in the serum of patients with glioma (n=82) and healthy controls $(n=42)$. (C) Overall survival and (D) disease-free survival of patients with high and low MMP-9 levels. (E) Overall survival and (F) disease-free survival of patients with high and low miR-34a. ${ }^{\# \#} \mathrm{P}<0.01$ vs. control group. Low expression of MMP9 was $<2$ of the MMP9 expression in healthy volunteers, high expression of MMP9 was $>2$ of the MMP9 expression of healthy volunteers; low expression of miR-34a was <2 of miR-34a the expression of healthy volunteers, high expression of miR-34a was $>2$ of the miR-34a expression of healthy volunteers. MMP-9, matrix metalloproteinase-9; Control, healthy volunteer group; miR, microRNA.

high MMP-9 expression were decreased compared with those with low MMP-9 expression. In addition, the OS and DFS of patients with low miR-34a expression were decreased compared with those with high miR-34a expression (Fig. 1E and F).

miR-34a regulates MMP-9 protein expression in glioma cells. To evaluate whether miR-34a regulated the protein expression of MMP-9 in glioma cells, a RT-qPCR and western blotting were used to analyze miR-34a and MMP-9 expression in U251-MG glioma cells transfected with miR-34a mimics and anti-miR-34a. As presented in Fig. 2A-C, the miR-34a mimic significantly increased miR-34a expression and significantly suppressed MMP-9 protein expression in glioma cells, compared with the negative control group $(\mathrm{P}<0.01)$. As presented in Fig. 2D-F, anti-miR-34a administration significantly decreased miR-34a expression and significantly induced MMP-9 protein expression in glioma cells, compared with the negative control group $(\mathrm{P}<0.01)$. Furthermore, it was demonstrated that miR-34a targeted the 3'-UTR of MMP-9 protein (Fig. 2G), as the luciferase activity of miR-34a was significantly inhibited in glioma cells, compared with the negative control group $(\mathrm{P}<0.01$; Fig. $2 \mathrm{H})$. Immunofluorescence demonstrated that overexpression of miR-34a significantly suppressed MMP-9 protein expression in glioma cells, in comparison with the negative control group $(\mathrm{P}<0.01$; Fig. 2I and J).
Downregulation of miR-34a promotes cell growth and migration, and inhibits apoptosis in glioma cells. To investigate the effects of miR-34a on the cell growth of glioma cells, anti-miR-34a was used. As presented in Fig. 3, downregulation of miR-34a significantly promoted cell growth (Fig. 3A), reduced LDH activity levels (Fig. 3B) and increased migration (Fig. 3C and D), significantly inhibited $\mathrm{L}$ and apoptosis (Fig. 3E and F), and significantly decreased caspase-3 and caspase-9 activity levels (Fig. 3G and $H$ ) in glioma cells, compared with the negative control group (all $\mathrm{P}<0.01)$.

Overexpression of miR-34a inhibits cell growth and migration, and induces apoptosis in glioma cells. To further determine the effects of miR-34a on cell growth of glioma cell, an miR-34a mimic was used in the present study. As presented in Fig. 4, overexpression of miR-34a significantly reduced cell growth and migration, significantly induced apoptosis and LDH activity levels, and significantly increased caspase-3 and caspase-9 activity levels in glioma cells, in comparison with the negative control group (all $\mathrm{P}<0.01$ ).

MMP-9 inhibitor attenuates the effects of miR-34a downregulation on glioma cells. SB-3CT (an inhibitor of MMP-9) was used to evaluate the role of MMP-9 in the effects 

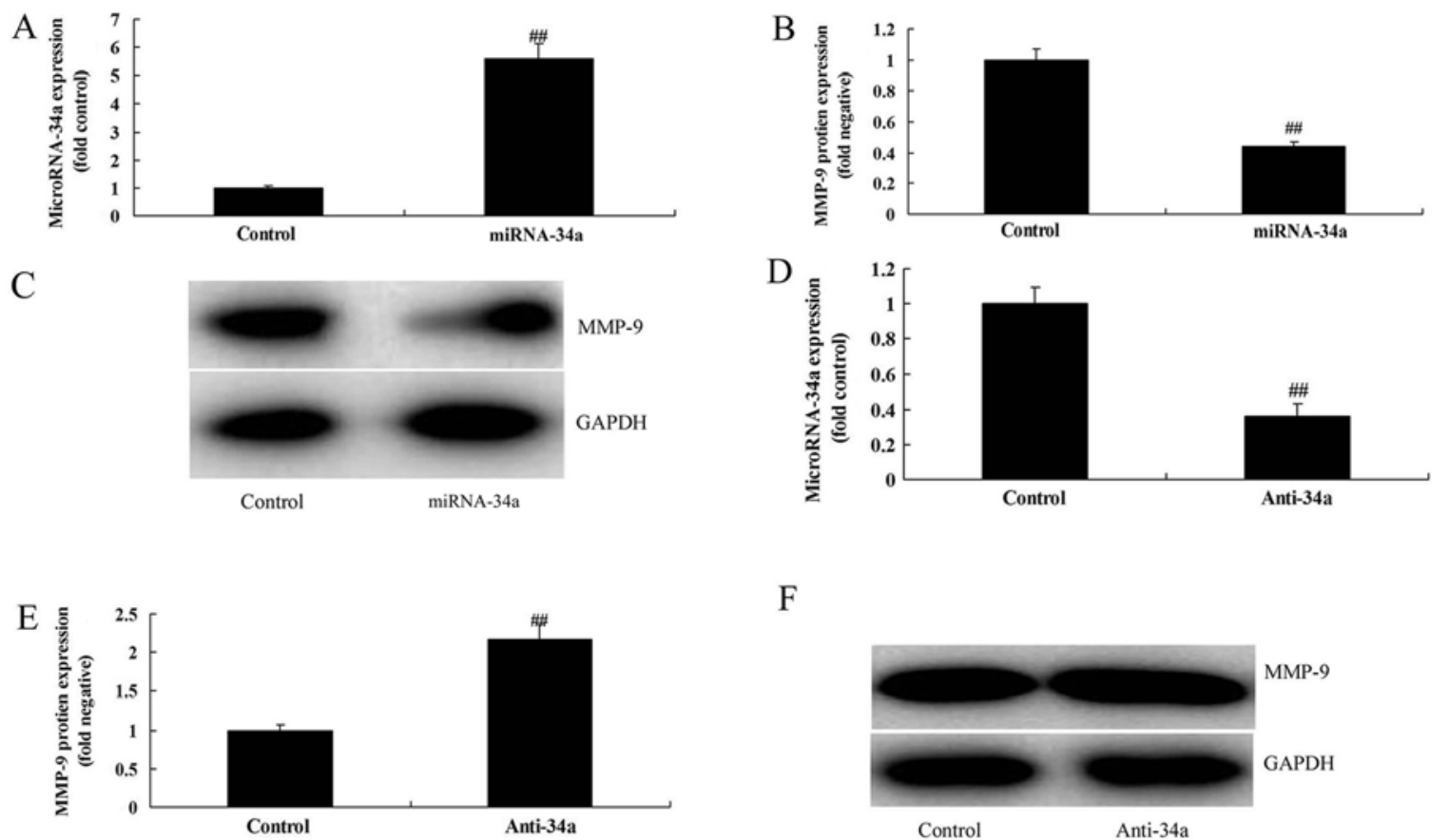

F

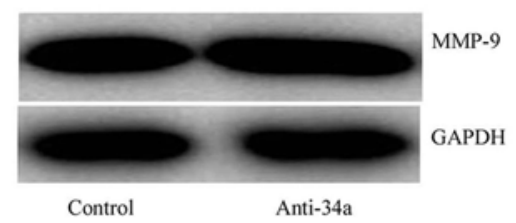

G
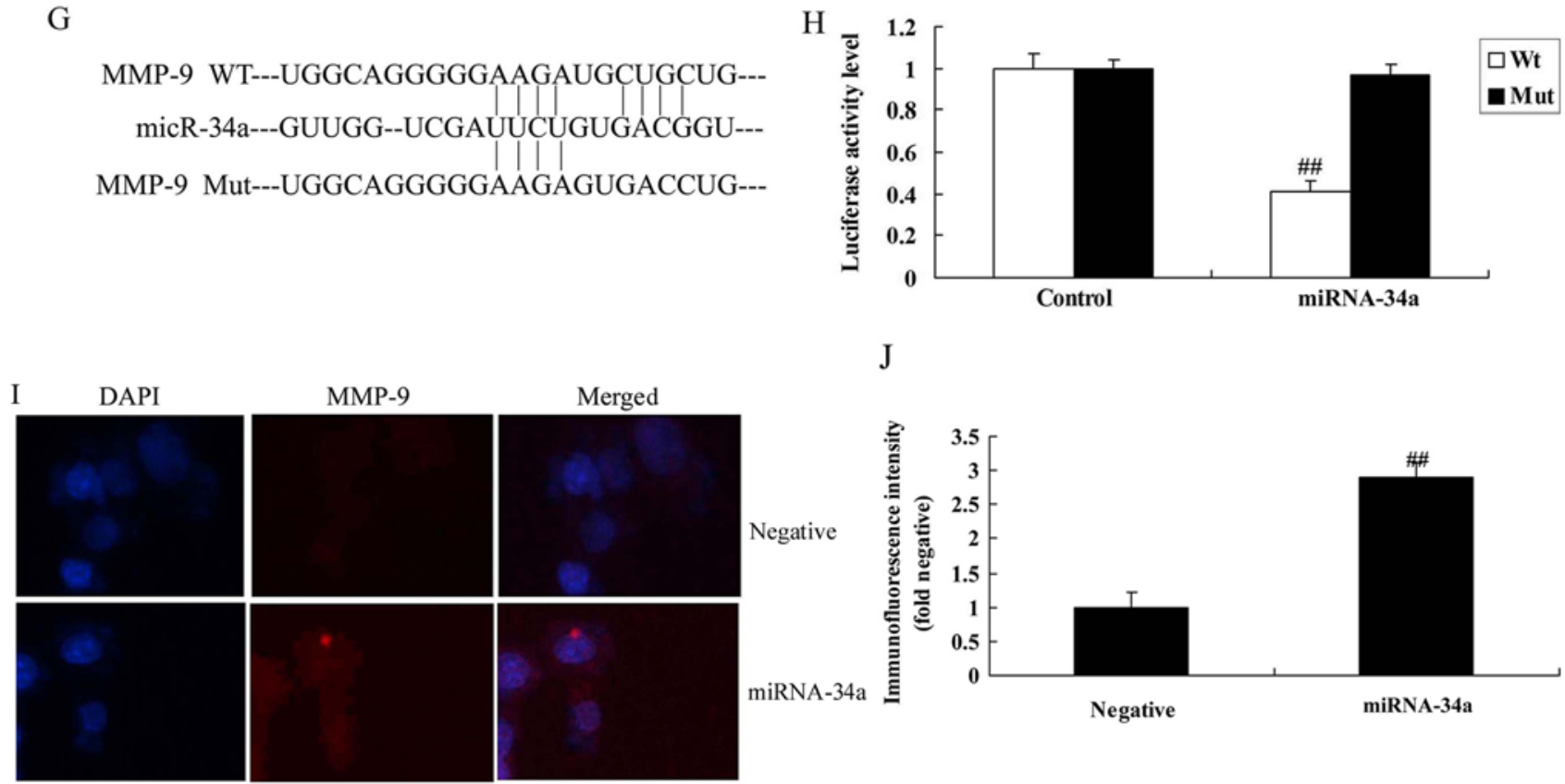

$\mathrm{J}$
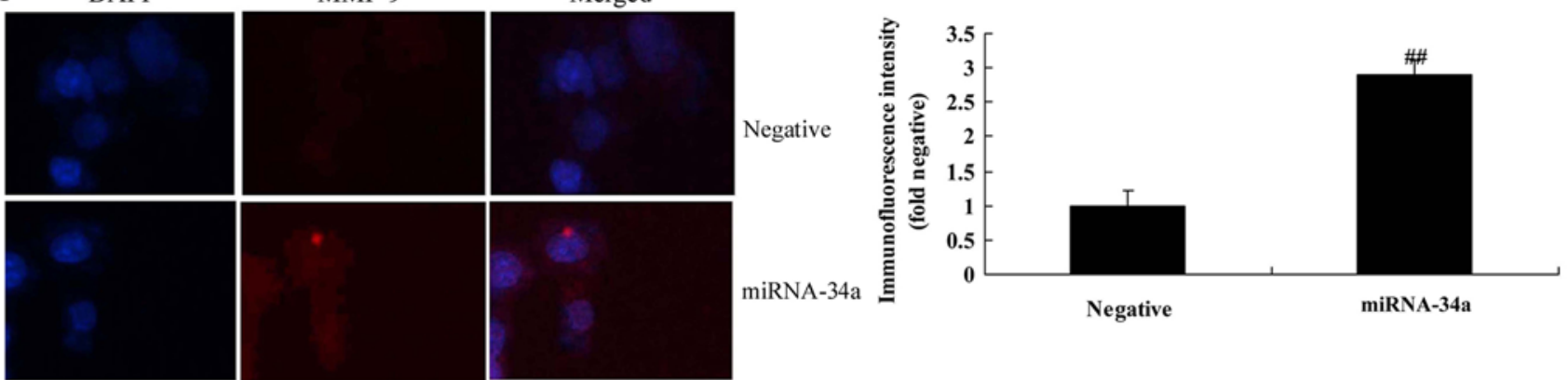

Figure 2. miRNA-34a regulates MMP-9 protein expression in glioma cells. U215 were transfected with miRNA-34a mimics and (A) miRNA-34a expression was determined by RT-qPCR, and (B) densitometry analysis of MMP-9 protein expression was determined by (C) western blotting. U215 were transfected with anti-34a and (D) miRNA-34a expression, (E) densitometry analysis of MMP-9 protein expression was determined by (F) western blot analysis. (G) miRNA-34a pairing with MMP-9 3'-untranslated region. (H) Luciferase assay results. (I) Immunofluorescence of MMP-9 protein expression and (J) analysis of the results of the immunofluorescence. ${ }^{\# \#} \mathrm{P}<0.01$ vs. control group. Control, negative group; miRNA-34a, overexpression of miRNA-34a group; Anti-34a, downregulation of miRNA-34a group. MMP, matrix metalloproteinase; miRNA, microRNA; WT, wild-type; Mut, mutant.

of miR-34a in glioma cells. As presented in Fig. 5A and B, the MMP-9 inhibitor (100 nM) significantly suppressed the protein expression of MMP-9 in glioma cells following miR-34a downregulation, compared with the anti-miR-34a group $(\mathrm{P}<0.01)$. The MMP-9 inhibitor attenuated the effects of miR-34a downregulation on cell growth and migration, apoptosis rate, LDH activity levels and caspase-3/9 activity level in glioma cells following miR-34a downregulation, in comparison with the miR-34a downregulation group (Fig. 5C-J).

\section{Discussion}

Glioma is the most common primary intracranial tumor in adults, which has a high malignant grade, high potential for recurrence and poor prognosis (13). The results of the present study suggest that MMP-9 expression was increased, and miRNA-34a was suppressed in the serum of patients with glioma, compared with the normal group. The OS and DFS of MMP-9 high-expression groups were decreased compared 


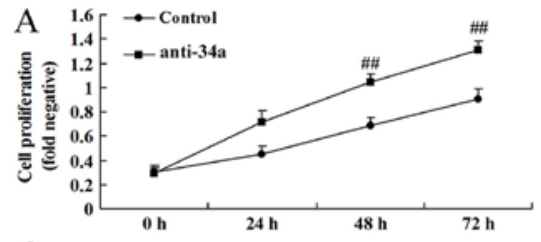

$\mathrm{C}$

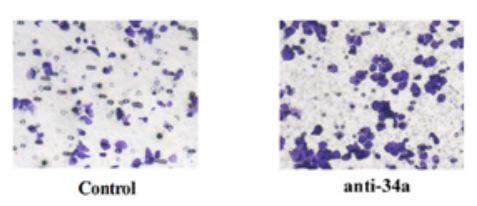

E
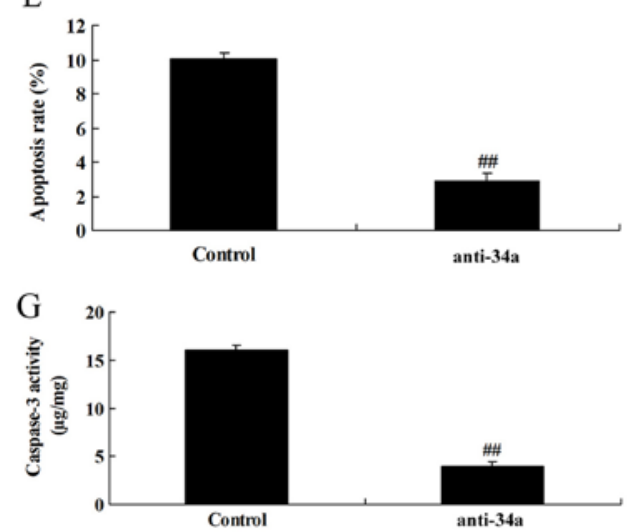
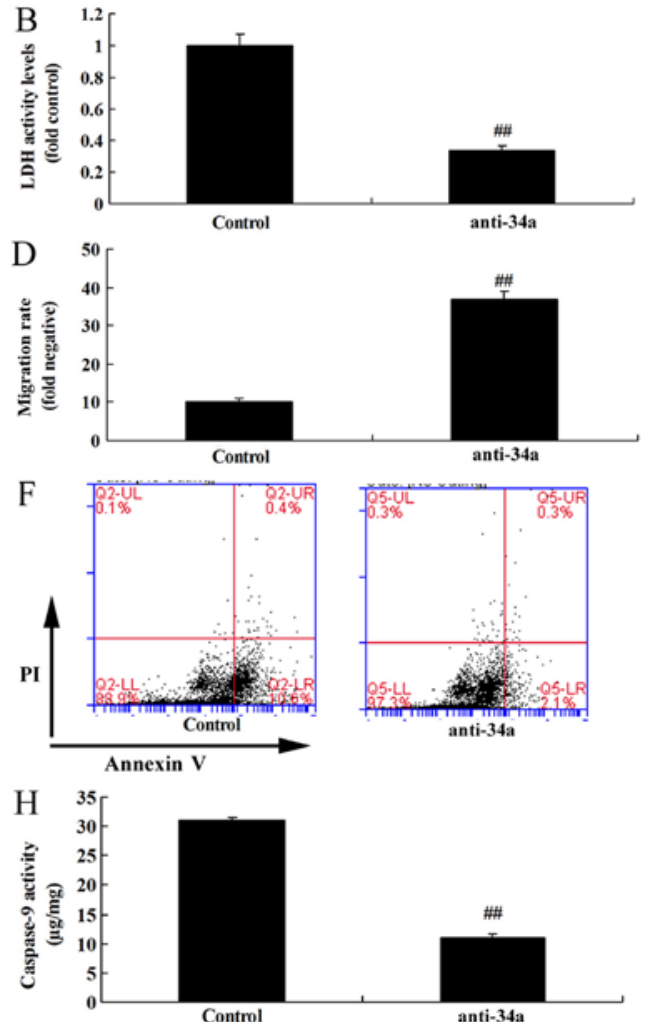

Figure 3. Downregulation of miRNA-34a promoted cell growth and migration, and inhibited apoptosis in glioma cells. (A) Cell growth, (B) LDH activity level, (C) Transwell migration assay (magnification, x100) and (D) migration rate, (E) apoptosis rate and (F) raw flow cytometry data, (G) caspase-3 and (H) caspase-9 activity. ${ }^{\# \#} \mathrm{P}<0.01$ vs. the control group. miRNA-34a, microRNA-34a; Control, negative group; anti-34a, miRNA-34a inhibitor; LDH, lactate dehydrogenase; PI, propidium iodide.

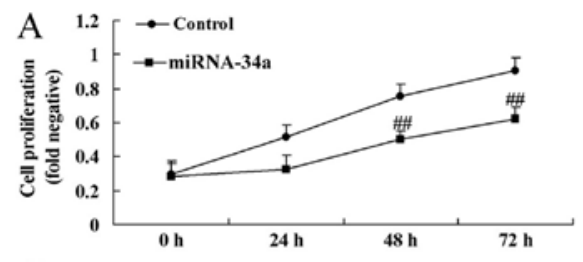

$\mathrm{C}$

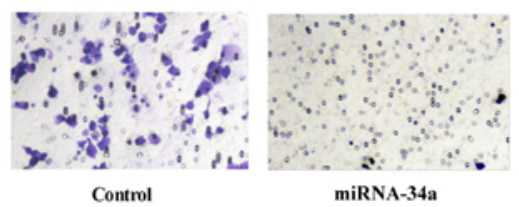

E
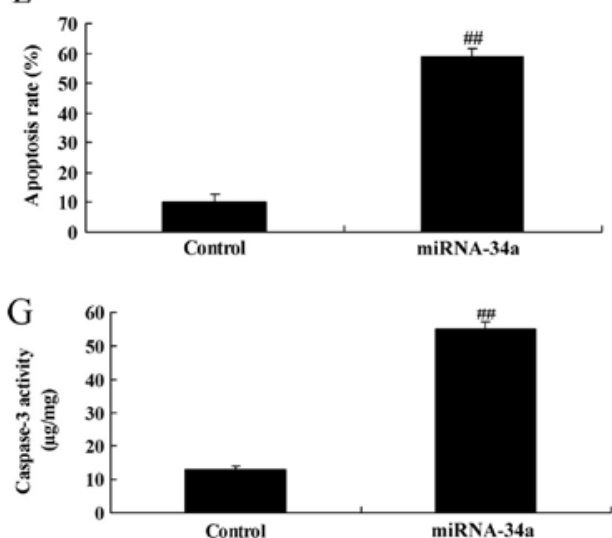
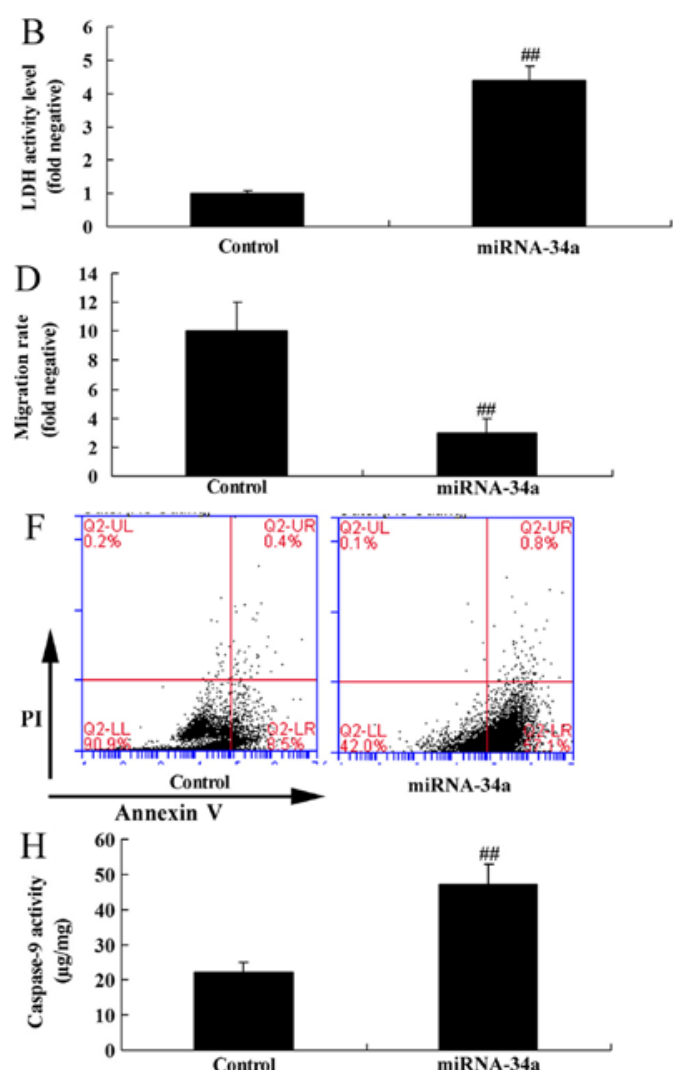

Figure 4. Overexpression of miRNA-34a inhibited cell growth and migration, and induced apoptosis in glioma cell. (A) Cell growth, (B) LDH activity level, (C) Transwell migration assay (magnification, x100) and (D) migration rate, (E) apoptosis rate and (F) raw flow cytometry data, (G) caspase-3 and (H) caspase-9 activity. ${ }^{\# \#} \mathrm{P}<0.01$ vs. control group. miRNA-34a, microRNA-34a; Control, negative group; miRNA-34a, miRNA-34a mimic overexpression; $\mathrm{LDH}$, lactate dehydrogenase; PI, propidium iodide. 
A

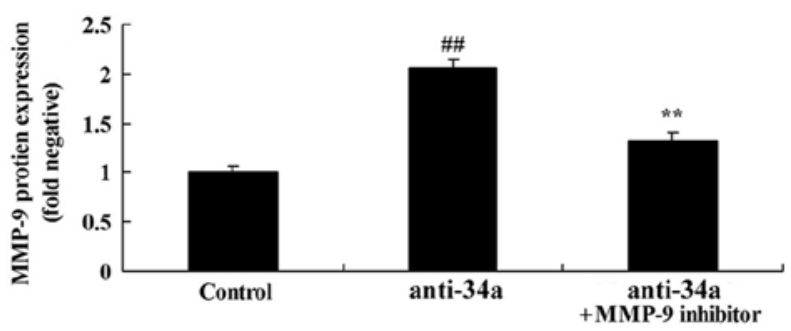

C

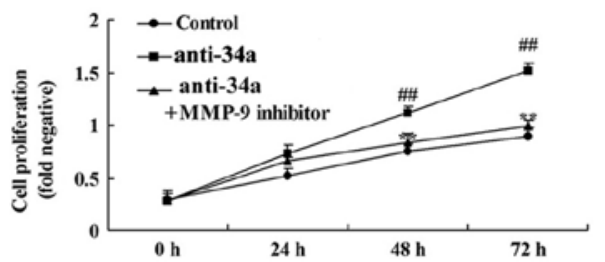

E

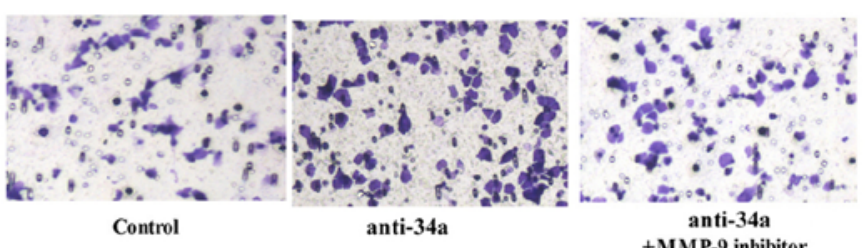

G
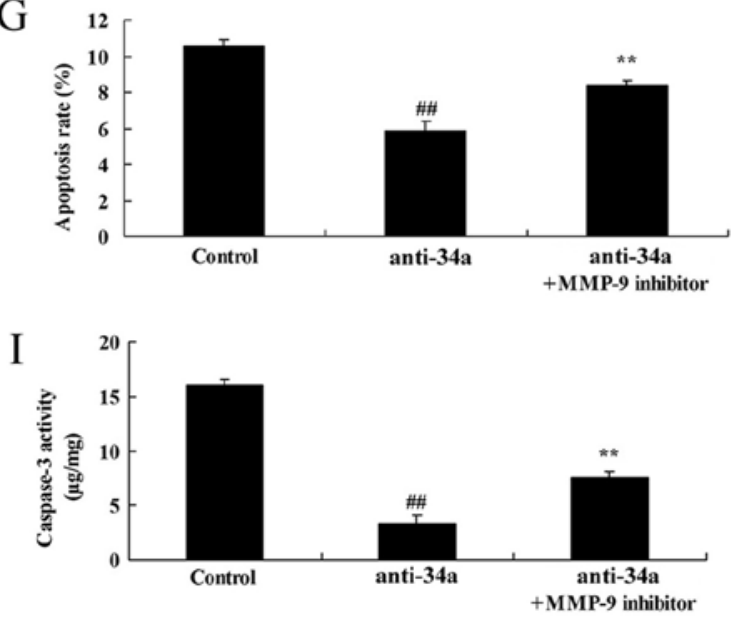

B

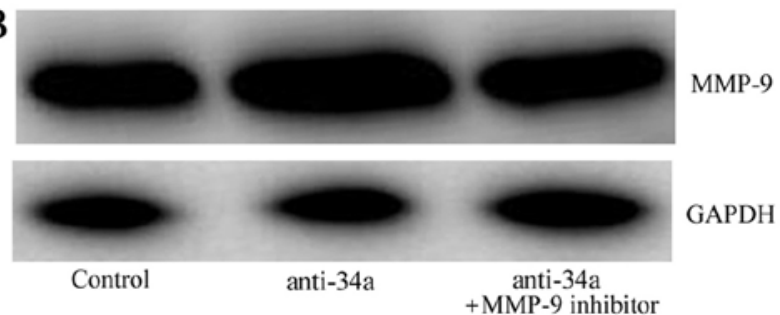

D

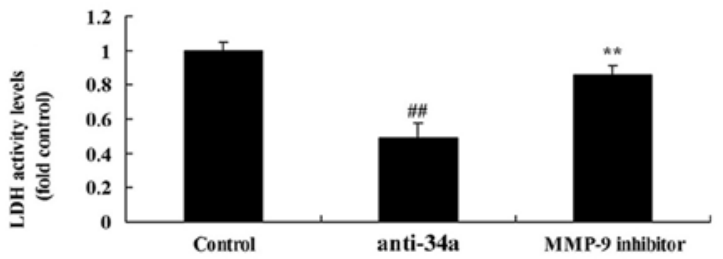

F

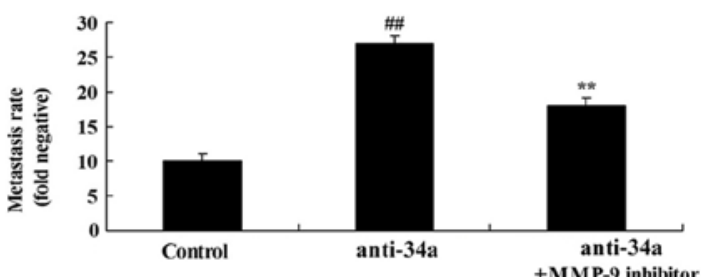

$\mathrm{H}$

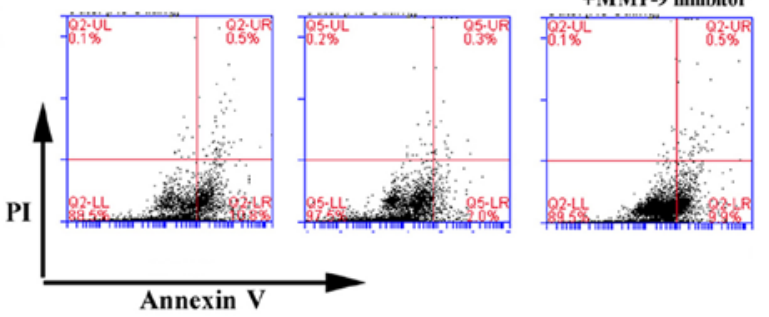

J

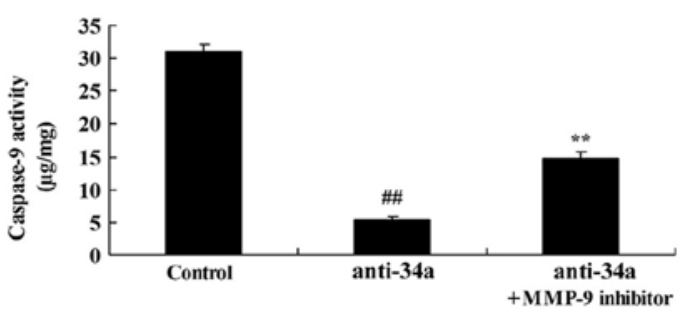

Figure 5. MMP-9 inhibitor reduced the effects of miRNA-34a downregulation in glioma cell. (A) MMP-9 protein expression using western blot analysis and (B) MMP-9 protein expression by statistical analysis, (C) cell growth, (D) LDH activity level, (E) Transwell migration assay (magnification, x100) and (F) migration rate, $(\mathrm{G})$ apoptosis rate analysis and $(\mathrm{H})$ flow cytometry data, (I) caspase-3 and $(\mathrm{J})$ caspase-9 activity and. ${ }^{\# \prime} \mathrm{P}<0.01$ vs. control; ${ }^{* *} \mathrm{P}<0.01$ vs. anti-34a. miRNA-34a, microRNA-34a; Control, negative group; anti-34a, miRNA-34a inhibitor; MMP-9 inhibitor, anti-34a + MMP-9 inhibitor (100 nM SB-3CT); MMP, matrix metalloproteinase; LDH, lactate dehydrogenase; PI, propidium iodide.

with the MMP-9 low-expression group. The OS and DFS of the miR-34a low-expression group were decreased compared with the miRNA-34a high-expression group. Overexpression of miR-34a significantly reduced cell growth and migration, and significantly increased apoptosis rates, LDH activity levels and caspase- 3 and caspase- 9 activity levels in glioma cells. In the present study, only one cell line U251-MG was used, which is insufficient and more cell models will be used in future studies.

MMPs are the endogenous zinc ion-dependent proteases essential for degrading the extracellular matrix (9). They can specifically degrade the basement membrane components. Gelatinases are the only MMP enzymes that can degrade the basement membrane and type IV collagen in the extracellular matrix. MMP-2 and MMP-9 are gelatinases that promote the break-through of tumor cells past the structural barrier of the basement membrane. In addition, these MMPs are associated with angiogenesis, and invasion and metastasis of tumors (9). However, in the present study, overexpression of miR-34a suppressed MMP-9 protein expression in glioma cells. Jia et al (11) reported that miR-34a reduced migration and invasion of tongue squamous cell carcinoma by targeting MMP-9 and MMP-14.

MMPs serve key roles during tumor metastasis by degrading multiple proteins in the extracellular matrix (14). Upregulated MMP expression in glioma can directly or indirectly degrade the extracellular matrix and basement membrane, which results in glioma invasion and peripheral tumor tissue edema (14). Consequently, MMPs are regarded as biomarkers of glioma progression (15). In addition, they can promote the structural rigidity, motility and proliferation of glioma cells (15). MMP inhibitors can suppress C6 glioma 
cell invasion in an optic nerve explant. Furthermore, MMP-2 and MMP-9 secreted by BT5C rat glioma cells can destroy cultured brain tissue. In addition, research in vitro suggests that MMPs secreted by glioma cells can decompose extracellular matrix proteins (14). Additionally, MMPs can promote tumor cell invasion into normal brain tissue, and enhance glioma invasion (14). In the present study, an MMP-9 inhibitor reduced the effects of miR-34a downregulation in glioma cells. Yang et al (16) indicated that miR-34a inhibits cell migration and invasion of esophageal squamous cell carcinoma by targeting MMP-2/MMP-9/fibronectin type III domain-containing protein $3 \mathrm{~B}$.

In conclusion, the present study demonstrated that MMP-9 expression was increased and miR-34a was suppressed in patients with glioma. The downregulation of miR-34a promoted cell growth and migration, and inhibited apoptosis in glioma cells by activation of MMP-9; thus enhancing the expression of miR-34a may be a novel strategy to suppress the progression of glioma.

\section{Acknowledgements}

Not applicable.

\section{Funding}

The present study was supported by funds from the Education Department of Jilin Province (grant no. JJKH20170056KJ).

\section{Availability of data and materials}

The analyzed data sets generated during the study are available from the corresponding author on reasonable request.

\section{Authors' contributions}

JP designed the study. XW, XC, LS, XB, HH and LC performed the experiments. JP and XW analyzed the data. JP wrote the manuscript.

\section{Ethics approval and consent to participate}

The present study was approved by the Ethics Committee of Affiliated Hospital of Beihua University. The study was performed in accordance with the regulations of the Institutional Review Board of Affiliated Hospital of Beihua University. Written informed consent was obtained prior to surgery from all enrolled patients.

\section{Patient consent for publication}

Written informed consent was obtained prior to surgery from all enrolled patients.

\section{Competing interests}

The authors declare they have no competing interests.

\section{References}

1. Wu XL, Huang H, Huang YY, Yuan JX, Zhou X and Chen YM: Reduced Pumilio-2 expression in patients with temporal lobe epilepsy and in the lithium-pilocarpine induced epilepsy rat model. Epilepsy Behav 50: 31-39, 2015.

2. Lin XT, Zheng XB, Fan DJ, Yao QQ, Hu JC, Lian L, Wu XJ, Lan P and He XS: MicroRNA-143 targets ATG2B to inhibit autophagy and increase inflammatory responses in crohn's disease. Inflamm Bowel Dis 24: 781-791, 2018.

3. He M, Zhan M, Chen W, Xu S, Long M, Shen H, Shi Y, Liu Q, Mohan M and Wang J: MiR-143-5p deficiency triggers EMT and metastasis by targeting HIF-1alpha in gallbladder cancer. Cell Physiol Biochem 42: 2078-2092, 2017.

4. Huang FT, Peng JF, Cheng WJ, Zhuang YY, Wang LY, Li CQ, Tang J, Chen WY, Li YH and Zhang SN: MiR-143 targeting TAK1 attenuates pancreatic ductal adenocarcinoma progression via MAPK and NF-kappaB pathway in vitro. Dig Dis Sci 62: 944-957, 2017.

5. Livak KJ and Schmittgen TD: Analysis of relative gene expression data using real-time quantitative PCR and the 2(-Delta Delta C(T)) method. Methods 25: 402-408, 2001.

6. Cui C and Shi X: miR-187 inhibits tumor growth and invasion by directly targeting MAPK12 in osteosarcoma. Exp Ther Med 14: 1045-1050, 2017.

7. Zhou W, Pal AS, Hsu AY, Gurol T, Zhu X, WirbiskyHershberger SE, Freeman JL, Kasinski AL and Deng Q: MicroRNA-223 suppresses the canonical NF-kappaB pathway in basal keratinocytes to dampen neutrophilic inflammation. Cell Rep 22: 1810-1823, 2018.

8. Chen M, Zhang H, Shi Z, Li Y, Zhang X, Gao Z, Zhou L, Ma J, Xu Q, Guan J, et al: The MST4-MOB4 complex disrupts the MST1-MOB1 complex in the Hippo-YAP pathway and plays a pro-oncogenic role in pancreatic cancer. J Biol Chem 293: 14455-14469, 2018.

9. Cheng J, Jing Y, Kang D, Yang L, Li J, Yu Z, Peng Z, Li X, Wei Y, Gong Q, et al: The role of Mst1 in lymphocyte homeostasis and function. Front Immunol 9: 149, 2018.

10. Huang Y, Huang H, Li M, Zhang X, Liu Y and Wang Y: MicroRNA-374c-5p regulates the invasion and migration of cervical cancer by acting on the Foxc1/snail pathway. Biomed Pharmacother 94: 1038-1047, 2017.

11. Jia LF, Wei SB, Mitchelson K, Gao Y, Zheng YF, Meng Z, Gan YH and Yu GY: miR-34a inhibits migration and invasion of tongue squamous cell carcinoma via targeting MMP9 and MMP14. PLoS One 9: e108435, 2014.

12. Tabouret E, Bertucci F, Pierga JY, Petit T, Levy C, Ferrero JM, Campone M, Gligorov J, Lerebours F, Roché $\mathrm{H}$, et al: MMP2 and MMP9 serum levels are associated with favorable outcome in patients with inflammatory breast cancer treated with bevacizumab-based neoadjuvant chemotherapy in the BEVERLY-2 study. Oncotarget 7: 18531-18540, 2016.

13. Martin-Alonso A, Cohen A, Quispe-Ricalde MA, Foronda P, Benito A, Berzosa P, Valladares B and Grau GE: Differentially expressed microRNAs in experimental cerebral malaria and their involvement in endocytosis, adherens junctions, FoxO and TGF-beta signalling pathways. Sci Rep 8: 11277, 2018.

14. Chen HX, Xu XX, Tan BZ, Zhang $Z$ and Zhou XD: MicroRNA-29b inhibits angiogenesis by targeting VEGFA through the MAPK/ERK and PI3K/Akt signaling pathways in endometrial carcinoma. Cell Physiol Biochem 41: 933-946, 2017.

15. Zhao Q, Ye M, Yang W, Wang M, Li M, Gu C, Zhao L, Zhang Z, Han W, Fan W and Meng Y: Effect of Mst1 on endometriosis apoptosis and migration: Role of drp1-related mitochondrial fission and parkin-required mitophagy. Cell Physiol Biochem 45: 1172-1190, 2018.

16. Yang L, Song X, Zhu J, Li M, Ji Y, Wu F, Chen Y, Cui X, Hu J, Wang L, et al: Tumor suppressor microRNA-34a inhibits cell migration and invasion by targeting MMP-2/MMP-9/FNDC3B in esophageal squamous cell carcinoma. Int J Oncol 51: 378-388, 2017.

This work is licensed under a Creative Commons Attribution-NonCommercial-NoDerivatives 4.0 International (CC BY-NC-ND 4.0) License. 